\title{
HISTOPLASMOSIS IN NON-HIV IMMUNOCOMPROMISED PATIENT RESIDING IN A NON-ENDEMIC AREA IN BRAZIL
}

Thatyana Siqueira Gonçalves ${ }^{1}$

Laura Pazinato Ritter ${ }^{2}$

Taiguara Fraga Guimarães ${ }^{3}$

Cássia Silva de Miranda Godoy, ${ }^{4,6}$

Renata de Bastos Ascenço Soares ${ }^{5,6}$

Abstract: Introduction: Histoplasmosis is a fungal disease, caused by Histoplasma capsulatum. Goal: To report a case of an immunocompromised patient with diagnosis of histoplasmosis in a non-endemic region. Methodology: Case report. Case report: Patient carrier of rheumatoid arthritis, makes continuous use of methotrexate and has reported contact with large amount of bat guano. Result of cervical lymph node culture was positive for $H$. capsulatum. Discussion: The infection was presented related to drug-induced immunosuppression in a non-endemic area. Conclusion: In view of the location where the infection occurred, the geographic expansion of the disease and the importance of this report for the literature are clear.

Key words: Histoplasmosis; Histoplasma capsulatum; Immunocompromised

\footnotetext{
${ }^{1}$ Graduada em Medicina Pontifícia Universidade Católica de Goiás (PUC Goiás) Goiânia GO - 74175-120, Brasil. thatysiqueeira@gmail.com, (i) https://orcid.org/0000-0002-4746-7615

${ }^{2}$ Graduada em Medicina Pontifícia Universidade Católica de Goiás (PUC Goiás) Goiânia GO - 74175-120, Brasil.

lauritter@gmail.com, iD https://orcid.org/0000-0001-8855-3424/print

${ }^{3}$ Médico Residente em Infectologia, Hospital das Clínicas, Universidade Federal de Goiás, Av, Universitária s/n., Setor Universitário Goiânia-GO, 74605-020, Brasil, taiguaramed@gmail.com, (i) https://orcid.org/0000-00017724-1038

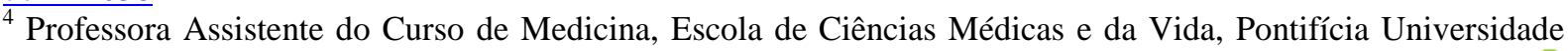
Católica de Goiás, Goiânia GO - 74175-120, Brasil. cassiamirandagodoy@hotmail.com, (D) https://orcid.org/0000-0003-4893-0541

${ }^{5}$ Professora Adjunta do Curso de Medicina, Escola de Ciências Médicas e da Vida, Pontifícia Universidade Católica de Goiás, Goiânia GO - 74175-120, Brasil. renatasuarezbastos@gmail.com, https://orcid.org/0000-0003-1029-325X

${ }^{\frac{1}{6}}$ Hospital Estadual de Doenças Tropicais Dr Anuar Auad, Alameda do Contorno, 3556 - Jardim Bela Vista, Goiânia - GO - 74850-400, Brasil.
}

\begin{tabular}{|c|cc|}
\hline Recebido em & Aceito em & Publicado em \\
\hline $23 / 08 / 2021$ & $06 / 10 / 2021$ & $13 / 10 / 2021$ \\
\hline
\end{tabular}




\section{INTRODUCTION}

Histoplasmosis is a systemic mycosis caused by Histoplasma capsulatum. It is endemic in North America and, in the United States; is one of the most common mycoses, with predominance in the areas around the valleys of the rivers Ohio and Mississippi (1). In Canada, histoplasmosis is considered endemic in the regions bordering the São Lourenço River, especially Quebec (2).

Being an endemic disease in South America, in Brazil several cases have been reported in the south, southeast and northeast regions (3). Most patients infected with $H$. capsulatum in Brazil are associated with Acquired Immunodeficiency Syndrome (AIDS). In contrast, the states of Rio Grande do Sul, Rio de Janeiro, São Paulo, Minas Gerais and Mato Grosso do Sul recorded a series of cases involving immunocompetent patients (4). Within the Northeast region, the state of Ceará stands out, with a large number of cases. In the Brazilian Midwest, in Goiás for example, there are no epidemiological studies on the disease, despite the existence of several medical reports (3).

H. capsulatum is characterized by a thermal dimorphism in which the filamentous form, which is infectious, requires a temperature change from $25^{\circ} \mathrm{C}$ to $37^{\circ} \mathrm{C}$ to become the parasite form of yeast, thus being able to survive in the intracellular environment. As for ecology, this species has nitrogen or phosphate enriched soils, associated with guano of birds or bats, as natural habitat (1). This relationship is due to the presence of high uric acid content in the feces of these animals, a source of nutrients for $H$. capsulatum, which causes them to be found in microenvironments such as caves, abandoned buildings, chicken coops, trees or any other places where soil can be enriched with feces from bats, chickens and other gregarious birds. Ideal conditions of soil temperature, humidity and $\mathrm{pH}$ are also necessary for the survival of this fungus (5).The infectious process can occur in two ways: the first one begins after inhalation of large amounts of micronids or small fragments of hyphae, while the second occurs after the reactivation of quiescent infected cells during immunosuppression (6). To enter the macrophages, $H$. capsulatum interacts with cellular receptors and, simultaneously, minimizes its recognition from signaling receptors by masking immunostimulatory molecules (7). Upon reaching the mediastinal lymph nodes, the lymphohematogenous spread to the liver, spleen, bone marrow, lungs and other organs begins, determining secondary foci. Cellular immunity is activated about 10 to 18 days after the primary infection constituting a granulomatous reaction that is followed by caseous 
necrosis, fibrous encapsulation and frequent deposition of calcium salts in the residual lesions (5).

\section{Clinical condition}

Histoplasmosis presents itself in several syndromes, which vary according to the clinical course, extent and radiological findings. Among them are subacute pulmonary histoplasmosis, acute pulmonary histoplasmosis, chronic pulmonary histoplasmosis, and disseminated histoplasmosis.

Disseminated histoplasmosis is defined by the presence of extra-pulmonary and mediastinal extraganglionic focus of progressive course. In this condition, the association of mycosis with Hodking disease, lymphosarcoma, leukemia, systemic lupus erythematosus, AIDS or any other condition of depression of cellular immunity is very common, as in individuals transplanted or using immunosuppressive therapy, especially in endemic areas (5)

Among the most common clinical manifestations are fever, fatigue, malaise, anorexia, weight loss and respiratory symptoms. Physical examination often reveals lymphadenopathy, hepatosplenomegaly, oral and cutaneous lesions depending on the extra-pulmonary area involved. $(8,9)$.

\section{Diagnosis}

The culture is still considered gold standard for the diagnosis of histoplasmosis; however, this method is time-consuming, requiring at least 15 days, besides having low sensitivity (10). Among them are immunological tests to detect antibodies and / or antigens and, more recently, molecular techniques to identify $H$. capsulatum (11). Immunodiagnostic tests are based on two methods of antibody detection - complement fixation (CF) and immunodiffusion (ID) - that are useful not only for diagnosis but also for patient monitoring.

Antigen detection is the most useful serological method for the diagnosis of disseminated histoplasmosis in patients with AIDS. Currently, it is of major importance for the detection of urinary antigen, both for diagnosis and therapeutic monitoring. The sensitivity was $98 \%$, and the specificity of the UAg test was $99 \%$ (12). 


\section{Treatment}

Clinical severity guides the choice of therapeutic regimen. Thus, clinical forms are classified as mild to moderate and moderate to severe, when there are signs indicating pancytopenia, CNS impairment, hemodynamic instability and organic dysfunctions, including respiratory failure (13). In the mild to moderate form of the disease, the host immunocompetent or with symptoms less than one month does not need treatment. The therapeutic regimen recommended for the immunocompromised host or with symptoms for more than one month begins with Itraconazol $200 \mathrm{mg}$ three times a day for three days, followed by Itraconazol $200 \mathrm{mg}$ twice daily for 6-12 weeks (13). On the other hand, disseminated histoplasmosis in its moderate to severe form is treated with liposomal Amphotericin-B $3 \mathrm{mg} / \mathrm{kg} /$ day for 1-2 weeks, followed by Itraconazol $200 \mathrm{mg}$ three times a day for three days, then Itraconazol $200 \mathrm{mg}$ twice a day for at least 12 months (13).

The purpose of this manuscript is to report a case of an immunocompetent patient with diagnosis of histoplasmosis in a non-endemic region.

\section{METHODS}

Case report of Histoplasmosis in non-HIV immunocompromised patient residing in a non-endemic area in Brazil. Informed consent form was signed. Data were collected from medical records and supplemented with scientific articles.

\section{CASE REPORT}

Day 0: Day of hospitalization.A brown 44-year-old female, housewife, native of Crateus - Ceará, Brazil, living in Uruaçu - Goiás, Brazil, presented on day 0 at a referral hospital, with afternoon fever ranging from $37.8{ }^{\circ} \mathrm{C}$ and $39{ }^{\circ} \mathrm{C}$, associated with dry cough, nocturnal sweating and weight loss of $10 \mathrm{~kg}$ for 1 month. She also reported abdominal pain in the epigastrium and right hypochondrium, nausea, inappetence, altered bowel rhythm and asthenia. At the time of admission, hepatosplenomegaly was observed on previous abdominal computed tomography (CT).At the physical examination, the patient presented a regular state, anicteric, acyanotic, afebrile, eupneic and hydrated. Cardiovascular system with regular 2-stroke heart rhythm, with normal and no-murmuric sounds. She was found to have a heart rate of $150 \mathrm{bpm}$. 
Respiratory apparatus with physiological vesicular murmurs, without adventitious noises. A globular and flaccid abdomen, was revealed painful to palpation of the deep right hypochondrium, with liver $2 \mathrm{~cm}$ from the costal border and presence of hydro-air noises. The patient is a carrier of rheumatoid arthritis and makes continuous use of methotrexate. She reported contact with large numbers of bat guanos as swepting tree-lined streets in front of her residence around day -300.The patient's profile opened the range for several diagnostic hypotheses: lymphoma, pulmonary tuberculosis, visceral leishmaniasis and methotrexate poisoning, which were investigated during hospitalization by means of complementary tests. When hospitalized, even without definite focus, was initiated antibiotic therapy with cefepime on the suspicion of infection by Pseudomonas sp. in patients with previous immunosuppressive use.The laboratory results of day 0 revealed hemogram with indexes within the limits of normality and metabolic panel within the normal ranges, with the exception of C-reactive protein. The results are shown in Table 1.

\begin{tabular}{|c|c|}
\hline \multicolumn{2}{|c|}{ Table 1: Laboratory results of day zero. } \\
\hline \hline C - reactive protein & RESULTS \\
\hline Total Bilirubin & $19.28 \mathrm{mg} / \mathrm{dL}$ \\
\hline Direct Bilirubin & $1.50 \mathrm{mg} / \mathrm{dL}$ \\
\hline Indirect Bilirubin & $1.20 \mathrm{mg} / \mathrm{dL}$ \\
\hline ALT & $0.30 \mathrm{mg} / \mathrm{dL}$ \\
\hline AST & $119 \mathrm{U} / \mathrm{L}$ \\
\hline EAS & $100 \mathrm{U} / \mathrm{L}$ \\
\hline Uroculture & Normal \\
\hline Hepatitis B, HBsAg & Negative \\
\hline Hepatitis C, Anti HCV & Negative \\
\hline HIV & Negative \\
\hline Syphilis & Negative \\
\hline Leishmaniasis & Negative \\
\hline Toxiplasmosis Ig G & Negative \\
\hline Toxiplasmosis Ig M & $11.1 \mathrm{IU} / \mathrm{mL}$ \\
\hline Cytomegalovirus Ig G & $0.06 \mathrm{IU} / \mathrm{mL}$ \\
\hline Cytomegalovirus Ig M & $201.70 \mathrm{AU} / \mathrm{mL}$ \\
\hline Epstein Barr, Anti - VCA Ig G & $0.40 \mathrm{AU} / \mathrm{mL}$ \\
\hline Epstein Barr, Anti - VCA Ig M & 31.05 \\
\hline
\end{tabular}

Resection of right posterior cervical lymph node was performed on day +3 . On day +5 cultures were performed for blood bacteria and cervical lymph node with negative results. Culture for blood tuberculosis and induced sputum were negative, rapid molecular assay for tuberculosis in sputum induced with DNA for undetectable $M$. tuberculosis. The culture for fungus in the blood 
was negative whereas in the cervical lymph node it was positive for Histoplasma capsulatum. Computed tomography $(\mathrm{CT})$ of the thorax $($ day +7$)$ revealed fine interstitial infiltrates with micronodules presenting a random distribution. Multiple mediastinal lymph node enlargement in upper and lower paratracheal chains, periaortic and infracarinal measuring up to $2.1 \mathrm{~cm}$ on the largest axis (Figure 1). Abdomen CT scan (day +7) showed liver with slightly enlarged dimensions, slightly enlarged spleen, increased caliber and sinuosity of vessels in the splenic cord (Figure 2).

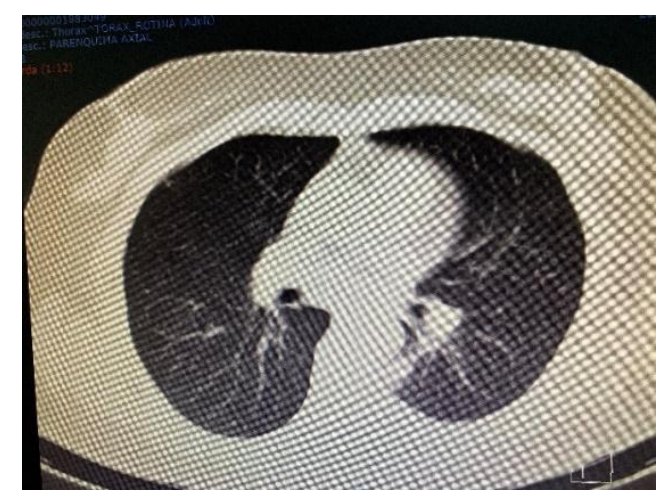

Figure 1. Chest computed tomography. Fine interstitial infiltrates with micronodules presenting random distribution. Multiple mediastinal lymph node enlargement in upper and lower paratracheal chains, periaortic and infracarinal measuring up to $2.1 \mathrm{~cm}$ on

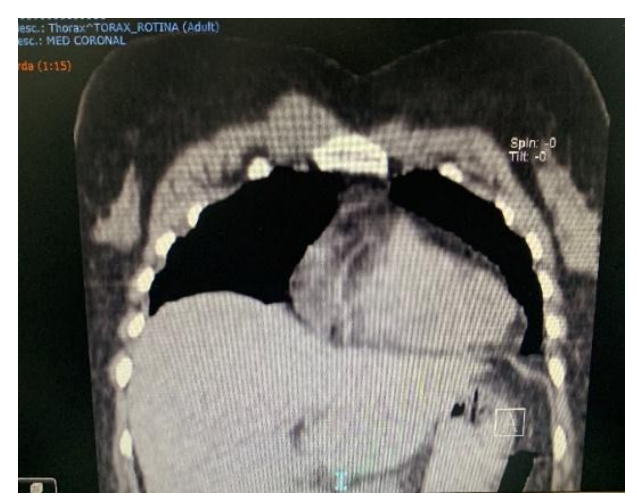

Figure 2. Computed tomography of the abdomen. Liver with slightly enlarged dimensions and slightly enlarged spleen, increased caliber and sinuosity of vessels in the splenic cord.

After the diagnosis of confirmed acute disseminated histoplasmosis and clinical improvement, the patient was discharged on day 16 on use of Itraconazol $200 \mathrm{mg}$ three times a day for 3 days. After this period, $200 \mathrm{mg}$ twice daily for 12 months and it's still under follow-up.

\section{DISCUSSION}

Histoplasmosis, in Brazil, predominates in the states of Ceará, Rio Grande do Sul, Mato Grosso do Sul and southeast region (4). Thus, this case draws attention to the fact that the patient is from non-endemic region, since she lives in Uruaçu, in the north of Goiás. Adjacent to the "Serra da Mesa" ridge, this municipality suffered a great environmental impact due to the flooding of caves inhabited by bats during the construction of a reservoir, provoking the search for refuge by these animals in rural and urban areas (14). Thus, the patient's exposure to a large 
number of bat guanos can be explained.

Some symptoms presented in the picture such as fever, weight loss and night sweats have led to the diagnostic suspicion of lymphoma and tuberculosis. Mycobacterial disease was also considered due to the cough and to the radiological pattern found in the tomography, characterized by multiple pulmonary nodules and mediastinal adenopathy (15). However, this diagnostic hypothesis was ruled out due to negative cultures and rapid molecular assay with DNA for undetectable $M$. tuberculosis. Leishmaniasis, on the other hand, had its diagnosis considered in view of the hepatosplenomegaly (16), but it was also ruled out because the serology for leishmaniasis was not reactive.

As the patient had continued use of methotrexate, a cytotoxic drug, and therefore, was immunosuppressed, severe or even fatal infections are part of the range of differential diagnoses. Thus, cough-associated fever may suggest pneumonia, since immunosuppressed individuals have recognized propensity for infection by opportunistic pathogens such as Pseudomonas aeruginosa (17). Thus, the patient of the case was submitted to the empirical antibiotic therapy with cephalosporin of 4th generation, which has high coverage for this pathogen.

The immunosuppression concomitant with exposure to a large amount of bat guanos is a risk factor for histoplasmosis (5), also supported by the clinical picture of fever, weight loss, nocturnal sweating, asthenia, cough and hepatosplenomegaly perceived at physical examination and confirmed by abdominal CT (9).

Elevation in liver lesion and function markers and the presence of reticulonodular and interstitial infiltrates on chest CT favored suspicion on histoplasmosis (9). This suspicion was confirmed by the culture of fungus positive for Histoplasma capsulatum in the cervical lymph node, which remains the gold standard for the diagnosis of this disease (10).

Taking into account the involvement of extrapulmonary foci demonstrated by hepatosplenomegaly on CT and mediastinal extra-ganglionic (cervical lymphonode) confirmed by biopsy, the disease was classified as disseminated (5).

After the diagnostic confirmation of disseminated histoplasmosis from mild to moderate type, standard treatment with Itraconazol was instituted, since the condition did not present neurological involvement, pancytopenia or hemodynamic instability (13). 


\section{CONCLUSION}

Although histoplasmosis is commonly associated with HIV-infected immunosuppressed patients, in this case it has been reported related to drug-induced immunosuppression. It is also worth mentioning that the infection occurred in a non-endemic area of South America, thus demonstrating the geographic expansion of the mycosis and confirming the importance of this case for the literature.

\section{REFERENCES}

1. Teixeira MDM, Patané JSL, Taylor ML, Gómez BL. Worldwide Phylogenetic Distributions and Population Dynamics of the Genus Histoplasma. PLoS Negl Trop Dis. 2016;1-20.

2. Brown EM, McTaggart LR, Dunn D, Pszczolko E, Tsui KG, Morris SK, et al. Epidemiology and geographic distribution of blastomycosis, histoplasmosis, and coccidioidomycosis, Ontario, Canada, 1990-2015. Emerg Infect Dis. 2018;24(7):1257-66.

3. Silva TC, Treméa CM, Zara ALSA, Mendonça AF, Godoy CSM, Costa CR, et al. Prevalence and lethality among patients with histoplasmosis and AIDS in the Midwest Region of Brazil. Mycoses. 2017;60(1):59-65.

4. Faiolla RCL, Coelho MC, Santana R de C, Martinez R. Histoplasmosis in immunocompetent individuals living in an endemic area in the Brazilian Southeast. Rev Soc Bras Med Trop. 2013;46(4):461-5.

5. Muniz M de M. Caracterização Molecular do Histoplasma capsulatum isolados no Brasil. 2009.

6. Passos AN, Kohara VS, Freitas RS De, Vicentini AP, Paulo S, Médica LDM, et al. Immunological assays employed for the elucidation of an histoplasmosis outbreak in São Paulo, SP. Brazilian J Microbiol. 2014;1361(4):1357-61.

7. Garfoot AL, Rappleye CA, State O. Histoplasma capsulatum surmounts obstacles to intracellular pathogenesis. FEBS J. 2017;283(4):619-33.

8. Hage CA, Azar MM, Bahr N, Loyd J, Wheat LJ. Histoplasmosis: Up-to-Date EvidenceBased Approach to Diagnosis and Management. Semin Respir Crit Care Med. 2015;36(5):729-45.

9. Nair U, Moorthy RS, Cunningham ET. Histoplasmosis. Intraocular Inflamm. 2016;30:1293-9.

10. Guimarães AJ, Nosanchuk JD, Zancopé-Oliveira RM. Diagnosis of histoplasmosis. 
Brazilian J Microbiol. 2006;37(1):1-13.

11. Scheel CM, Gómez BL. Diagnostic Methods for Histoplasmosis: Focus on Endemic Countries with Variable Infrastructure Levels. Curr Trop Med Reports. 2014;1(2):129-37.

12. Libert D, Procop GW, Ansari MQ. Histoplasma Urinary Antigen Testing Obviates the Need for Coincident Serum Antigen Testing. Am J Clin Pathol. 2018;149:362-368.

13. Sanford. Sanford Guide. 2019.

14. Fernandes CTC. Impactos Socioambientais de Grandes Barragens e Desenvolvimento: a percepção dos atores locais sobre a Usina Hidrelétrica de Serra da Mesa. 2010.

15. Singer-Leshinsky S. Pulmonary tuberculosis. J Am Acad Physician Assist [Internet]. 2016;29(2):20-5. Available from: http://www.ncbi.nlm.nih.gov/pubmed/26757063\%0Ahttp://content.wkhealth.com/linkback/ openurl?sid=WKPTLP:landingpage $\&$ an=01720610-201602000-00003

16. Press D. Leishmaniasis in humans : drug or vaccine therapy? Drug Des Dev Ther. 2018;25-40.

17. Migiyama Y, Yanagihara K, Kaku N, Harada Y, Yamada K, Nagaoka K, et al. Pseudomonas aeruginosa bacteremia among immunocompetent and immunocompromised patients: Relation to initialantibiotic therapy and survival. Jpn J Infect Dis. 2016;69(2):916. 